\title{
Conditional Deoxyribozyme-Nanoparticle Conjugates for miRNA-Triggered Gene Regulation
}

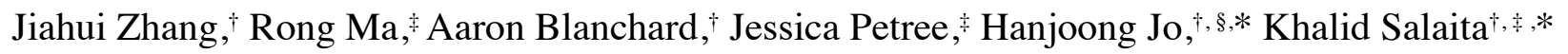

Wallace H. Coulter Department of Biomedical Engineering, Georgia Institute of Technology and Emory University, Atlanta, GA, USA

*Department of Chemistry, Emory University, Atlanta, GA, USA

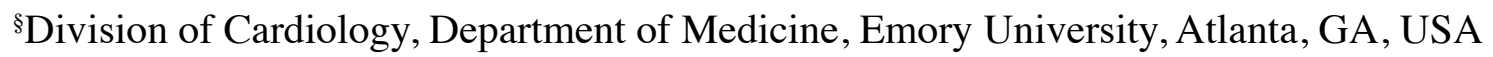

*Correspondence should be addressed to H.J. (email: hjo@emory.edu) or K.S. (email: k.salaita@emory.edu)

Note 1. Customized algorithm for prediction of mouse TNF $\alpha$ DNAzymes

Figure S1. Screen of a small library of DNAzymes that target mouse TNF $\alpha$ in RAW264.7 cells

Figure S2. Optimization of TNF $\alpha$ DNAzyme Dz-168

Figure S3. Activity of Dz strand and locked Dz compared to parental Dz-168

Figure S4. Dz locking is inefficient with unmodified toehold and branch migration domains in vitro

Figure S5. Exogenously transfected miR-33 upregulates TNF $\alpha$ expression

Figure S6. Characterization of AuNPs

Figure S7. Characterization of locked DzNPs with different toehold length

Figure S8. Characterization of DzNPs engineered with an anchored Dz strand

Figure S9. Uptake of locked DzNPs by RAW264.7 cells

Figure S10. Potential pathways of Dz activation and DNA degradation

Table S1. Oligonucleotide sequences

Table S2. Library of mouse TNF $\alpha$ DNAzymes screened

Table S3. Primer sequences 


\section{Note 1. Customized algorithm for prediction of mouse TNF $\alpha$ DNAzymes}

1. Selection of DNAzyme binding sites: Binding sites were selected using a software package custom written in MATLAB 2017a or later (Supplementary Software 1). The algorithm is illustrated in Figure S1a. First, the RNA sequence of interest (in this case, NM_000594.3 (human) or NM_001278601.1 (mouse)) was scanned from 5' to 3' for AU or GU junctions. At each AU or GU junction, the "target base" was defined as either the A or the G. Next, the free energy of hybridization $\left(\Delta G_{h y b}\right)$ of a 5 -base sequence immediately 5 ' to the target base (but not including the target base) was calculated according to the nearest-neighbor model presented by Sugimoto et al. ${ }^{1}$ using MATLAB's oligprops() function and $\mathrm{T}=37^{\circ} \mathrm{C}$ and [salt $]=0.015 \mathrm{M}$. The sequence length was increased incrementally until the calculated $\Delta G$ was below $-10 \mathrm{kcal} / \mathrm{mol}$, and all sequence lengths with $-8 \mathrm{kcal} / \mathrm{mol}>\Delta G_{h y b}>-10 \mathrm{kcal} / \mathrm{mol}$ were stored as potential "left arm" DNAzyme binding candidates. A similar process was then repeated to determine "right arm" binding candidates by evaluating the sequence immediately 3 ' to the target base. If both left arm and right arm binding candidates were found, then all combinations of left and right arms were concatenated (with the target base included between the two arms) and stored as potential DNAzyme target sequences.

2. Ranking of DNAzyme binding sites: DNAzyme target sequences were ranked by the free energy of their most stable secondary structure ( $\Delta G_{2^{\circ}}$ calculated using the rnafold() function in MATLAB with all default settings), which serves as an estimate for the target sequence's expected availability to DNAzyme binding. Target sequences with the same $\Delta G_{2^{\circ}}$ were then ranked based on the location of the target base as measured from the 5' end. Each target sequence was then converted to a DNAzyme sequence by taking the complement and replacing the target base with the sequence of the DNAzyme core. A ranked list of all DNAzyme sequences including human and mouse gene target base locations, left and right arm lengths, and $\Delta G_{2^{\circ}}$ was then stored to a .xlsx file (Supplementary Spreadsheet 1) for distribution. 


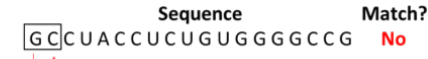

GCCUACCUCUGUGGGGCCG No

GCCUACCUCUGUGGGGCCG Yes

2) Optimize left arm binding energy (-8 to $-10 \mathrm{kcal} / \mathrm{mol}$ )

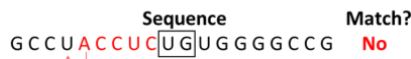

GCCUACCUCUGUGGGGCG No

GCCUACCUCUGUGGGGCG Yes

b

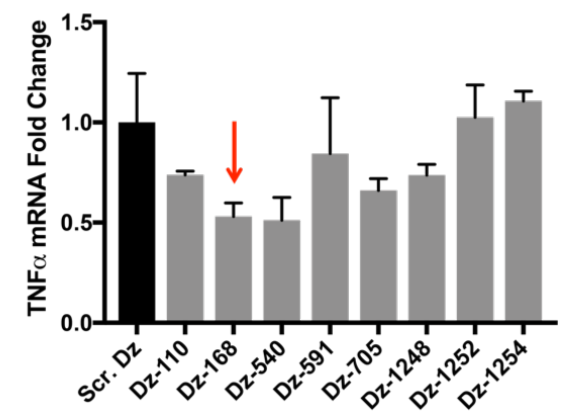

C

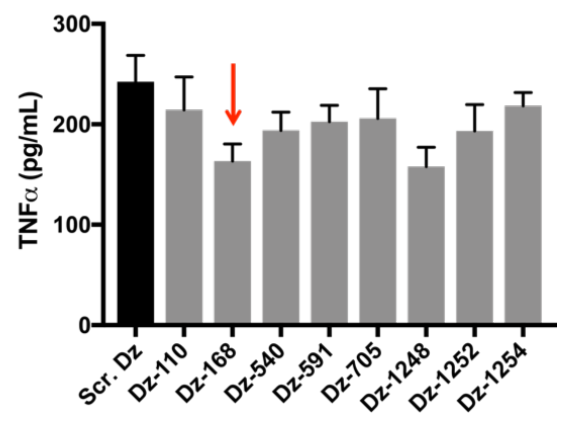

Figure S1. Screen of a small library of DNAzymes that target mouse TNFa in RAW264.7 cells. (a) Schematic describing the computational DNAzyme selection process. First, the mRNA sequence is scanned for target sites and, at each target site, arm length is tuned until each arm has a free energy of hybridization within a specified range. Target sequences are then ranked by secondary structure free energy and stored. This process is repeated for the entire mRNA sequence and multiple DNAzymes can be obtained for a given target site. (b, c) RAW264.7 cells were transfected with $200 \mathrm{nM}$ of each DNAzyme using Oligofectamine, and incubated for $24 \mathrm{~h}$. A non-specific DNAzyme (NS Dz) was included as a negative control. The standard manufacturer recommended concentration of Oligofectamine was used for this screen $(2 \mu \mathrm{L} /$ well for 24 well plate). Each well was plated with $\sim 100,000$ cells. (b) RNA was isolated using RNease kit (QIAGEN \#74104), and TNF $\alpha$ mRNA levels were quantified by qRT-PCR. The primers are listed in Table S3. (c) The cell medium was collected for ELISA analysis of secreted TNF $\alpha$ using a commercial ELISA kit (Invitrogen \#88-7324-22). Sequences for each DNAzyme are included in Table S2. The error bars represent SEM of triplicate samples. The red arrow corresponds to the DNAzyme used in this work. 
a

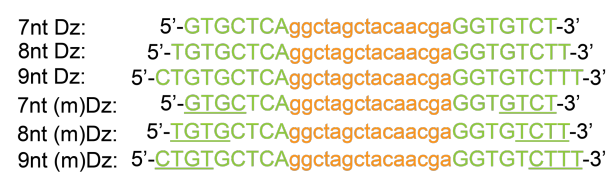

C

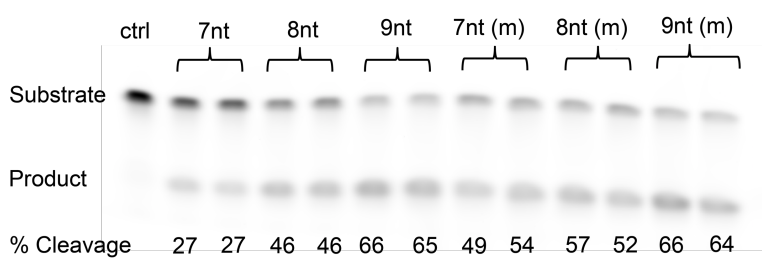

b

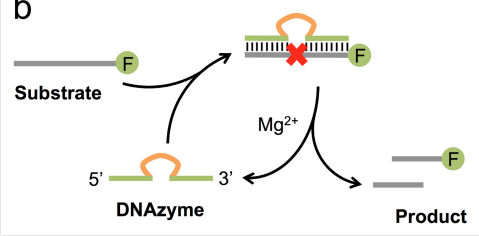

d

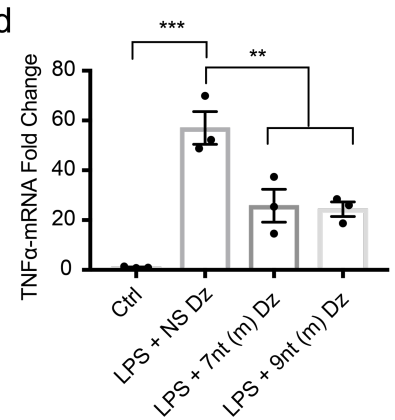

Figure S2. Optimization of TNF $\alpha$ DNAzyme Dz-168. (a) Sequences of mouse TNF $\alpha$ DNAzyme (Dz168) with different arm lengths and with or without 2'-OMe modifications. The green color corresponds to the left and right arms of the Dz that are complementary to the start codon region of mouse TNF $\alpha$ transcript. The lowercase orange sequence is the 10-23 catalytic domain of the Dz. The underlined nucleotides are 2'-OMe modified. (b) Schematic of substrate cleavage assay used to quantify catalytic activity of DNAzymes. (c) DNAzyme catalyzed multiple turnover of substrate cleavage. $200 \mathrm{nM}$ of DNAzymes were incubated with $1 \mu \mathrm{M}$ FAM-labeled substrates in $50 \mathrm{mM}$ Tris- $\mathrm{HCl}$ supplemented with $150 \mathrm{mM} \mathrm{NaCl}$ and $2 \mathrm{mM} \mathrm{MgCl}_{2}$ with $\mathrm{pH}$ 7.4. After incubation in a water bath at $37^{\circ} \mathrm{C}$ for $2 \mathrm{~h} 20 \mathrm{~min}$, the reaction mixture was mixed with the same volume of gel loading buffer and subjected to $15 \%$ MiniPROTEAN ${ }^{\circledR}$ TBE-Urea Gel. The gel was run with $170 \mathrm{~V}$ in $1 \times$ TBE buffer and imaged with an Amersham Typhoon Biomolecular Imager using the FITC channel. The \% cleavage values were determined using ImageJ analysis after background subtraction. (d) Plot showing TNF $\alpha$ levels in peritoneal macrophages transfected with non-specific (NS), 7nt(m) and 9nt(m) DNAzyme at a concentration of $1 \mu \mathrm{M}$. $24 \mathrm{~h}$ after transfection, $0.5 \mathrm{ng} / \mathrm{mL}$ lipopolysaccharide (LPS) was added, and cells were incubated for another $4 \mathrm{~h}$ before RNA isolation and qPCR analysis of TNF $\alpha$ mRNA level. The error bars represent SEM and each dot represents a biological replicate $\left(* * p<0.01\right.$, $*^{* *} p<0.001$, one-way ANOVA with Tukey's multiple comparison). 


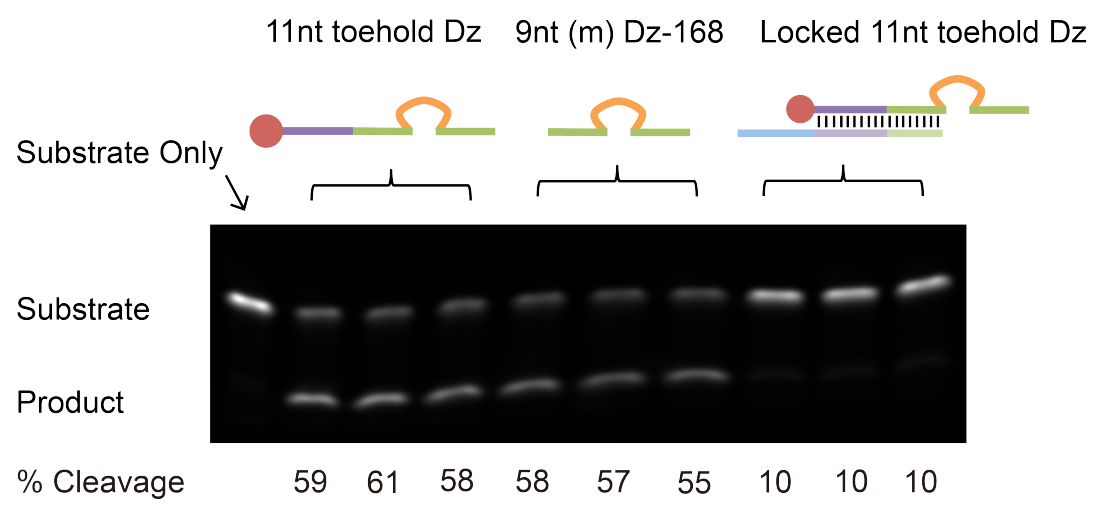

Figure S3. Activity of Dz strand and locked Dz compared to parental Dz-168 (9nt modified arms). To quantify Dz activity, $200 \mathrm{nM} 11 \mathrm{nt}$ toehold Dz, parental Dz-168, and locked 11 nt toehold Dz were incubated with $1 \mu \mathrm{M}$ FAM-labeled substrate in $50 \mathrm{mM}$ Tris- $\mathrm{HCl}$ supplemented with $150 \mathrm{mM} \mathrm{NaCl}$ and 2 $\mathrm{mM} \mathrm{MgCl}{ }_{2}$ with $\mathrm{pH} 7.4$ at $37{ }^{\circ} \mathrm{C}$ for $3 \mathrm{~h}$. The reaction mixture was then subjected to $15 \%$ MiniPROTEAN ${ }^{\circledR}$ TBE-Urea Gel. The first lane only includes substrate. The next three lanes were replicates of the $11 \mathrm{nt}$ toehold Dz, followed by three replicates of the parental Dz-168, and finally by the $11 \mathrm{nt}$ toehold Dz hybridized to the lock strand. The \% cleavage values were determined using ImageJ analysis after background subtraction. The sequences are shown in Table S2. 
A: Inactive Dz

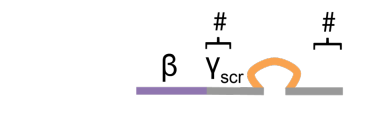

B: Locked Dz

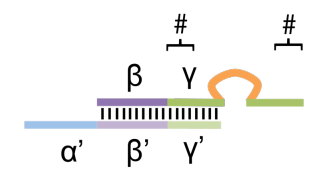

C: Active Dz

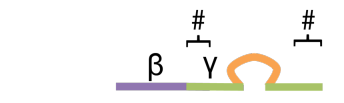

\# = 2'-OMe modification
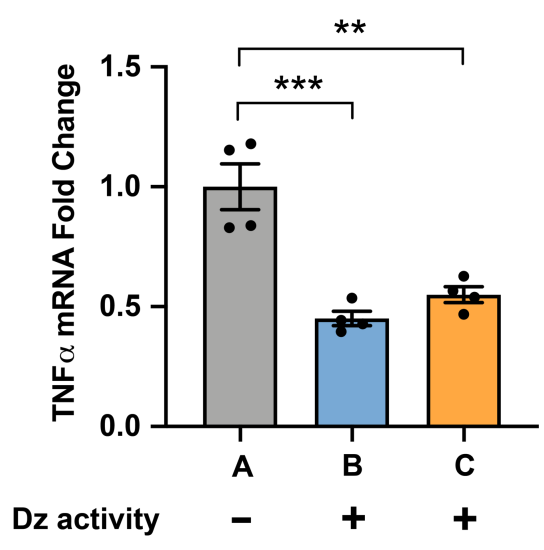

Lock strand - $\quad+\quad-$

Figure S4. Dz locking is inefficient with unmodified toehold and branch migration domains in vitro. RAW264.7 cells were transfected with inactive Dz (A), locked Dz with toehold (B) and unlocked Dz (C) using Oligofectamine and incubated for $24 \mathrm{~h}$. TNF $\alpha$ mRNA level was quantified by qRT-PCR. Dz activity: “-” indicates Dz with scrambled Dz binding arms, "+” indicates Dz with TNF $\alpha$ mRNA complementary binding arms; Lock strand: "-" indicates that the Dz is not hybridized to the lock strand, "+" indicates that the Dz is hybridized to the lock strand. The error bars represent SEM of biological replicates $(* * p<0.01$, $* * * p<0.001$, one-way ANOVA compared to A with Tukey's multiple comparison). 

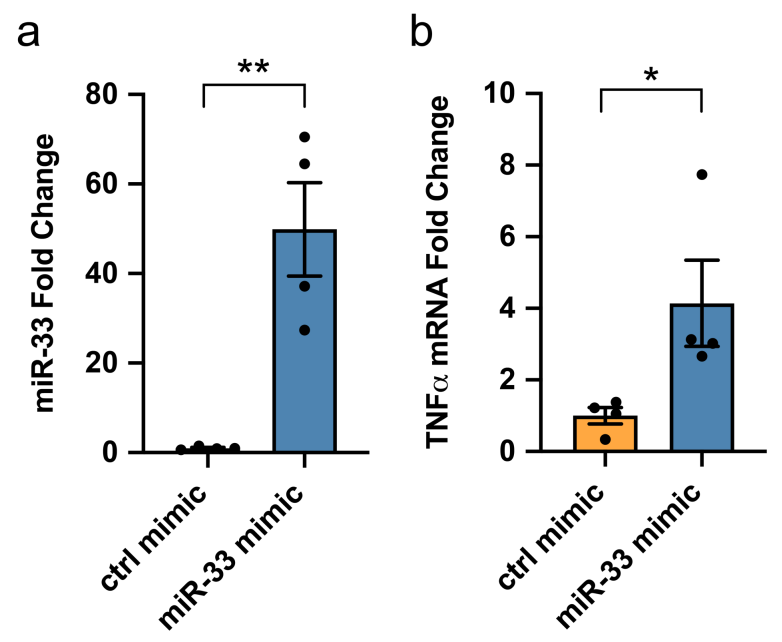

Figure S5. Exogenously transfected miR-33 upregulates TNF $\alpha$ expression. RAW264.7 cells were transfected with $200 \mathrm{nM}$ mirVana ${ }^{\mathrm{TM}}$ miR-33 mimic (\#4464066) or mirVana ${ }^{\mathrm{TM}}$ negative ctrl mimic (\#4464058) using Oligofectamine. $24 \mathrm{~h}$ later, cells were transfected with $200 \mathrm{nM}$ locked Dz, and incubated for another 24h before RNA isolation and qRT-PCR analysis of (a) miR-33 and (b) TNF $\alpha$ mRNA. The error bars represent SEM for biological replicates $(* p<0.05$, $* * p<0.01$, two-tailed $t$ test). 
a

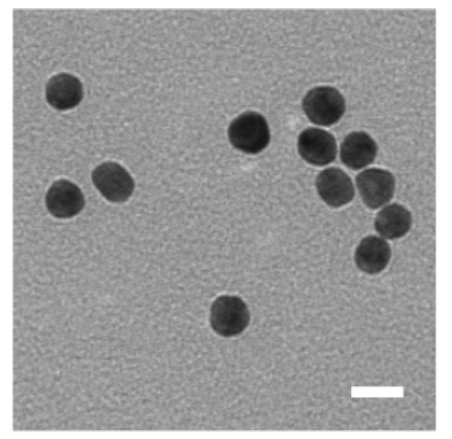

b

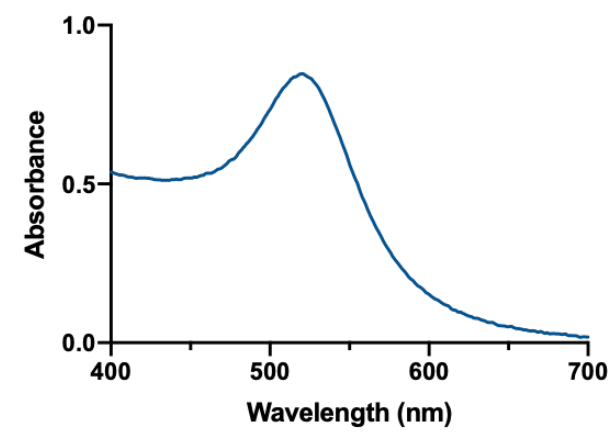

Figure S6. Characterization of AuNPs. (a) Representative TEM image (scale bar=20 nm), and (b) absorption spectrum of AuNPs used in this work. 
$11 \mathrm{nt}$ toehold

a

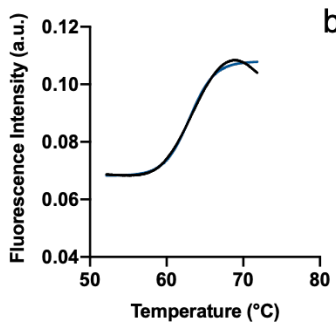

d

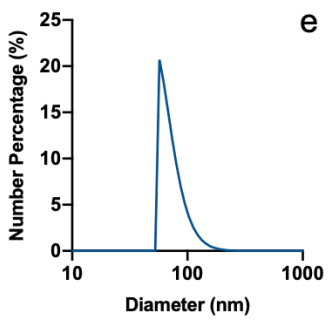

9

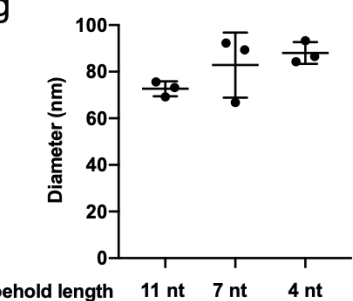

e
7 nt toehold
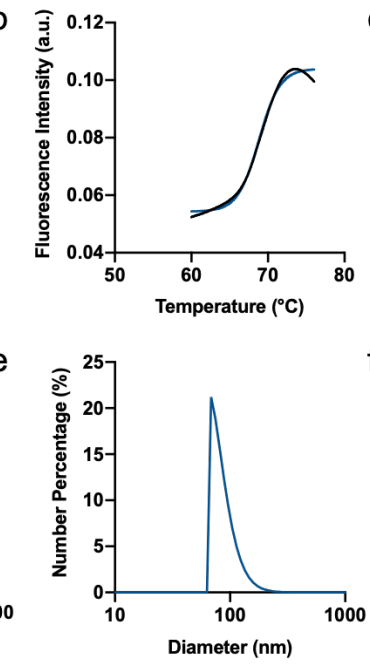

$\mathrm{h}$

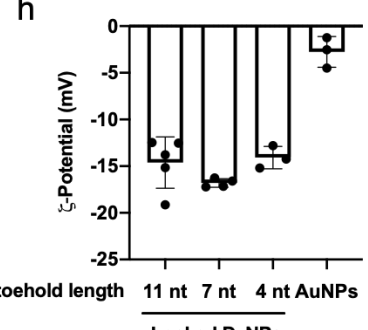

4 nt toehold

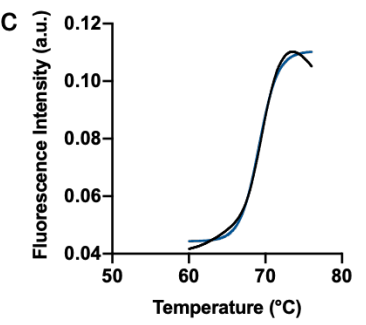

f

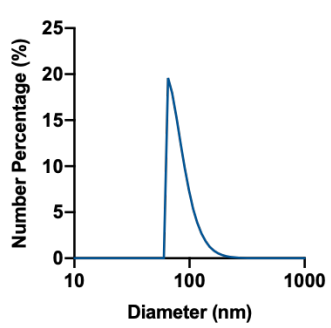

Locked DzNPs

Figure S7. Characterization of locked DzNPs with different toehold length. (a-c) Representative melting curves of locked DzNPs with (a) 11nt, (b) $7 \mathrm{nt}$ and (c) $4 \mathrm{nt}$ toehold. The fluorescence intensity of $5 \mathrm{nM}$ Cy5-labeled locked DzNPs with different toehold length in PBS was measured with a LightCycler ${ }^{\circledR} 96$ instrument as a function of temperature. The temperature was ramped from $45^{\circ} \mathrm{C}$ to $95^{\circ} \mathrm{C}$ at the rate of $0.04{ }^{\circ} \mathrm{C} / \mathrm{s}$, and 25 measurements were performed per ${ }^{\circ} \mathrm{C}$ with an interval of $0.04{ }^{\circ} \mathrm{C}$. The black lines indicate measured data, and the blue lines indicate fitted curves. $T_{\mathrm{m}}$ was determined as the temperature that generates a half-maximal fluorescence increase in the fitted curves. The data shown in Figure $4 \mathrm{~d}$ was compiled from three independent melts collected for each locked DzNPs. (d-f) Size distribution of locked DzNPs with (d) 11nt, (e) $7 \mathrm{nt}$ and (f) $4 \mathrm{nt}$ toehold measured by dynamic light scattering (NanoPlus zeta/nano particle analyzer, Particulate Systems). (g) Hydrodynamic diameters of locked DzNPs with different toehold length. (h) $\zeta$-potentials of locked DzNPs with different toehold length, as well as citrate-stabilized AuNPs, measured with the NanoPlus zeta/nano particle analyzer. The error bars represent SD. 
a

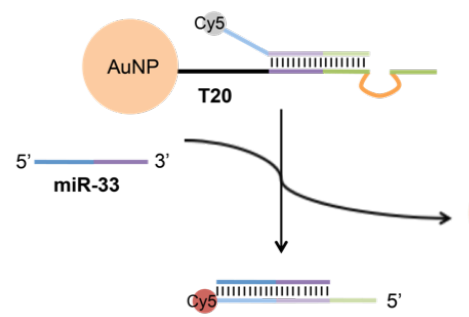

b

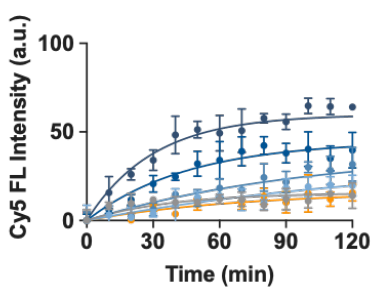

d

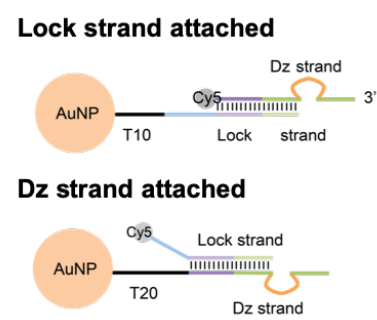

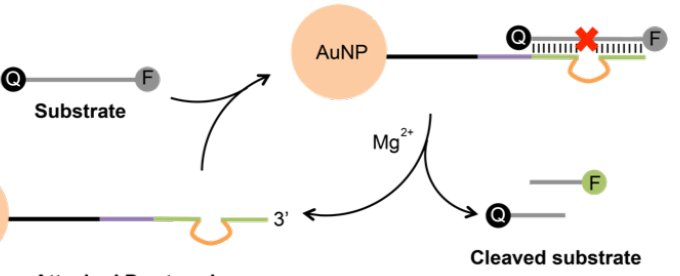

Attached Dz strand

C
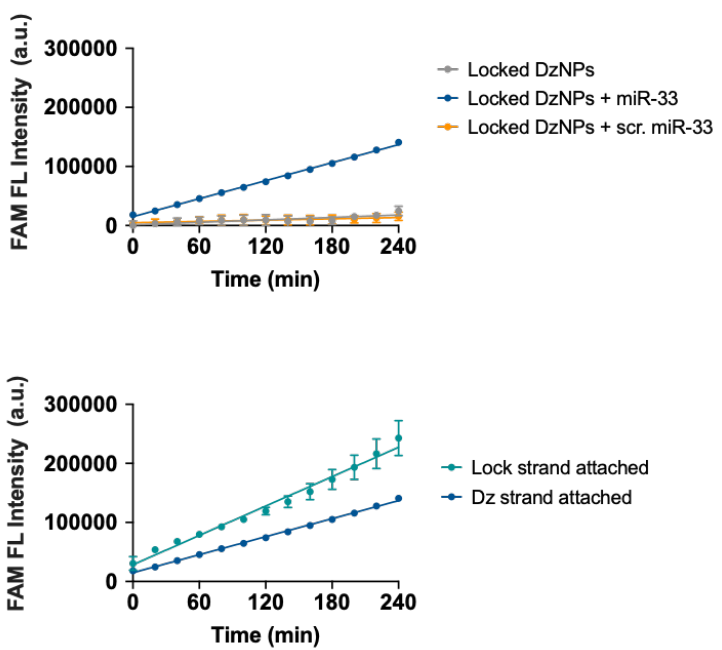

Figure S8. Characterization of DzNPs engineered with an anchored Dz strand. (a) Scheme for Dz strand anchored locked DzNPs. (b) Cy5 fluorescence intensity of $0.5 \mathrm{nM}$ locked DzNPs incubated with different concentrations $(0,5,20,50,200,500 \mathrm{nM})$ of miR-33 trigger and $500 \mathrm{nM}$ scrambled miR-33 for $2 \mathrm{~h}$. The Cy5 fluorescence intensity quantifies the efficiency of toehold exchange as a function of time. The error bars represent SD of three replicates. (c) $0.5 \mathrm{nM}$ locked DzNPs was pre-incubated with $500 \mathrm{nM}$ miR-33 trigger or scrambled miR-33. After a $1 \mathrm{~h}$ incubation, $300 \mathrm{nM}$ of fluorophore-quencher tagged substrate was added and the fluorescence of FAM was measured for $4 \mathrm{~h}$. (d) Comparison of activities of $0.5 \mathrm{nM}$ locked DzNPs of two configurations incubated with $500 \mathrm{nM}$ miR-33 trigger. The plot shows the average intensity from three replicates, and the error bar represent SD and some of them were too small to show on the plot. 

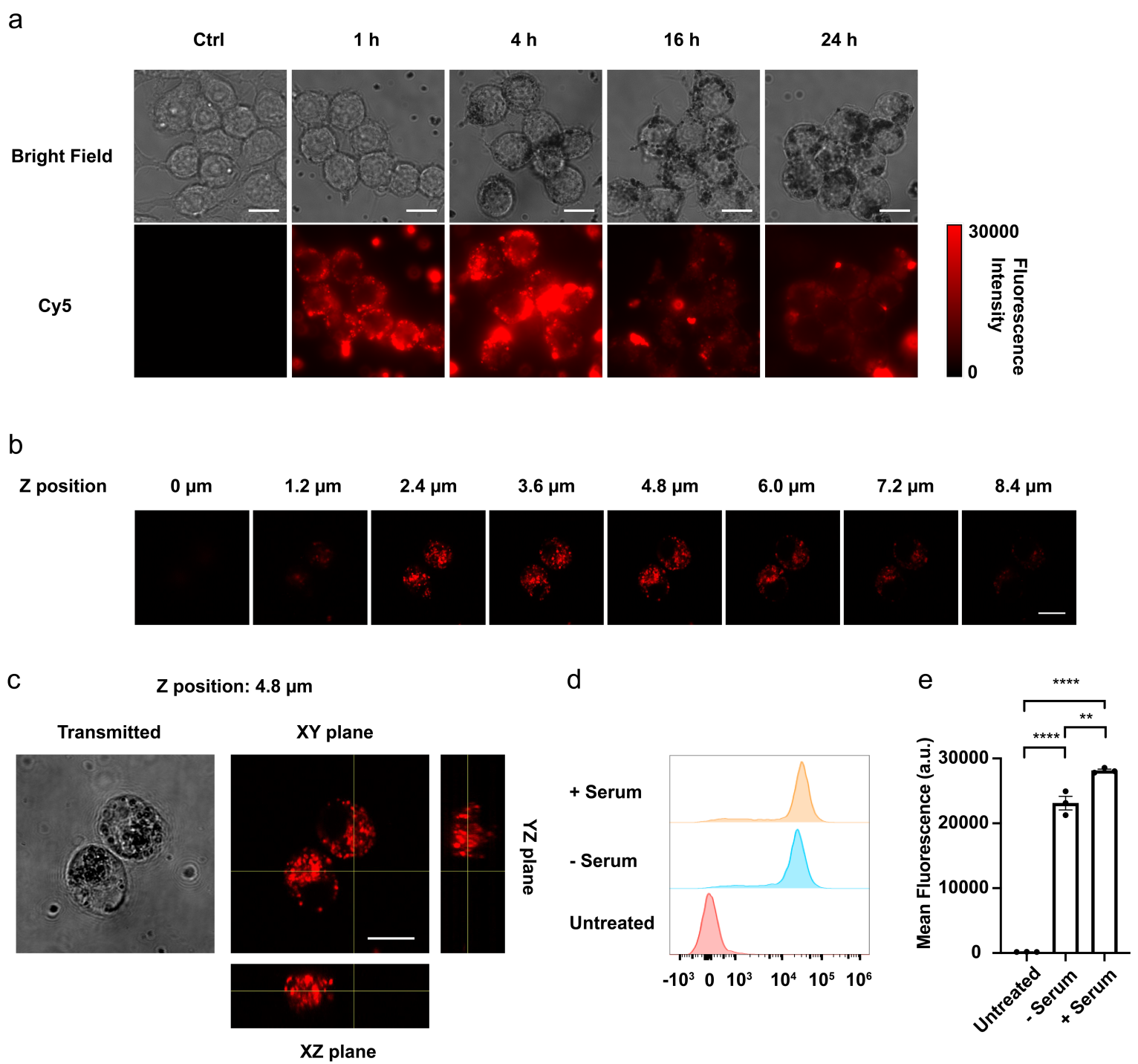

Figure S9. Uptake of locked DzNPs by RAW264.7 cells. (a) $5 \mathrm{nM}$ of 11 nt toehold locked DzNPs (with Cy-5 labeled Dz strands) were incubated with RAW264.7 cells for 1h, 4h, 16h and 24h. Cell uptake was assessed with widefield fluorescence imaging in the Cy5 channel. Scale bar represents $10 \mu \mathrm{m}$. (b, c) Confocal microscopy images of RAW264.7 cells incubated for 4h with $5 \mathrm{nM} 11 \mathrm{nt}$ toehold locked DzNPs (with Cy-5 labeled Dz strands). (b) Confocal images at different $\mathrm{Z}$ positions and (c) orthogonal view showing XY, YZ, and YZ planes when Z position was set to $4.8 \mu \mathrm{m}$. Scale bar represents $10 \mu \mathrm{m}$. (d,e) Flow cytometry analysis of RAW264.7 cells incubated for $4 \mathrm{~h}$ with $5 \mathrm{nM}$ of the $11 \mathrm{nt}$ toehold locked DzNPs (with Cy-5 labeled Dz strands) in the presence or absence of serum. Flow cytometry was performed to quantify DzNP uptake. (d) Representative histograms of cell-associated fluorescence and (e) mean fluorescence intensity, for untreated cells and cells that were incubated with DzNPs without (-) or with (+) serum. The error bars represent SEM for biological replicates. Each data point represents the mean fluorescence intensity of 4000 to 13000 cells in one replicate. $(* * p<0.01$, $* * * * p<0.0001$, one-way ANOVA with Tukey's multiple comparison). 


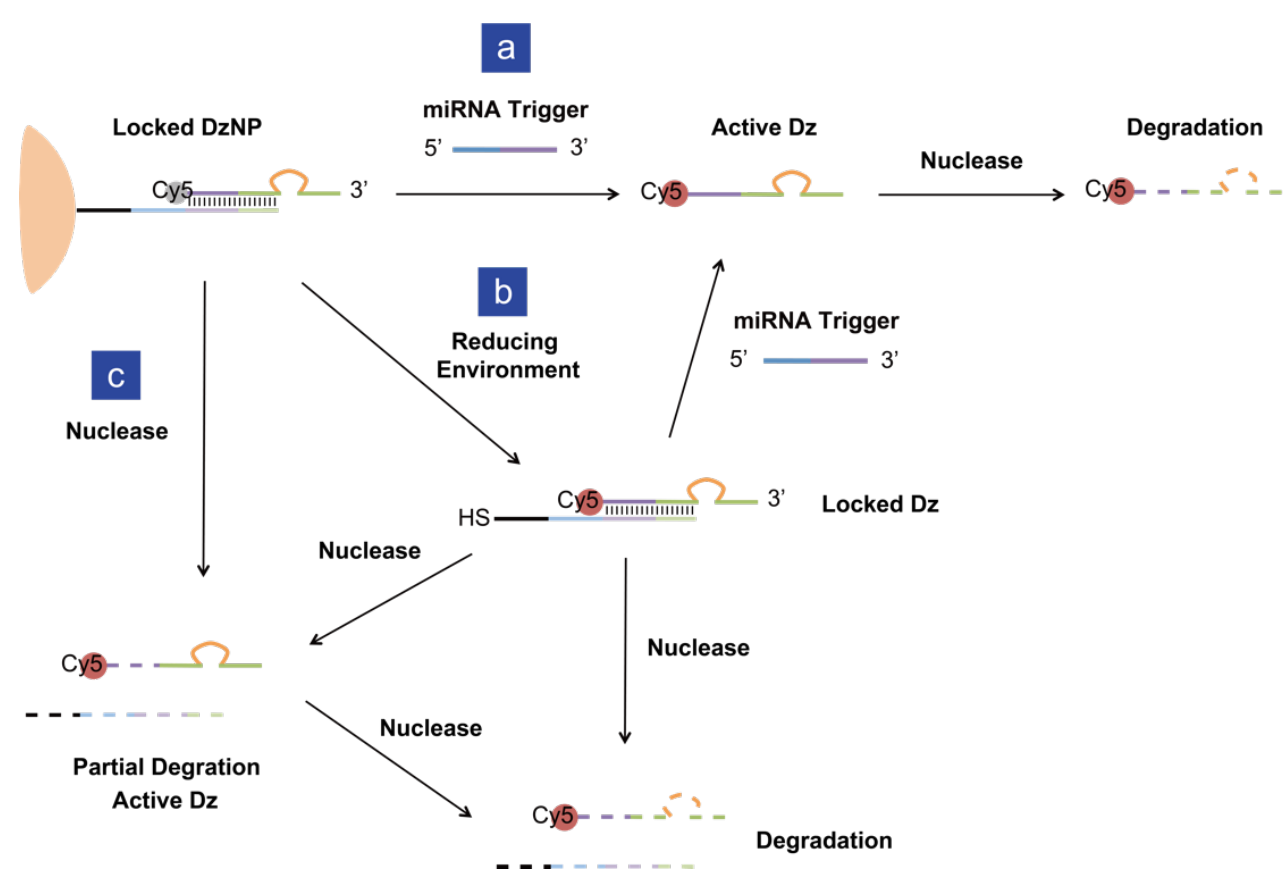

Figure S10. Potential pathways of Dz activation and DNA degradation. (a) Dz strand is displaced from the lock strand while the lock strand is attached on the AuNPs; (b) DNA duplex detachment from the AuNP surface due to thiol-exchange, followed by Dz strand displacement and activation; and (c) Locked $\mathrm{Dz}$ is partially degraded and released from AuNP surface, which causes background activation if the Dz is not completely degraded. Eventually, all DNA is degraded and Dz activity is eliminated due to the activity of celluar nucleases. 
Table S1. Oligonucleotide sequences

BHQ = Black Hole Quencher; FAM =6-Carboxyfluorescein; m=2'-O-Methyl modification; r=RNA base; /5AmMC6/=5' Amino Modifier C6; /3ThioMC3-D/=3' Thiol Modifier C3 SS; /5ThioMC6-D/=5' Thiol Modifier C6 SS; $/ 3 \mathrm{AmMO} /=5$ ' Amino Modifier

\begin{tabular}{|c|c|}
\hline Name & Sequence $\left(5^{\prime} \rightarrow 3^{\prime}\right)$ \\
\hline miR-33 trigger & GTG CAT TGT AGT TGC ATT GCA \\
\hline Scrambled miR-33 & AGC TTG ATG TTC GTT AGG CAT \\
\hline $7 \mathrm{nt} \mathrm{mDz}$ & $\begin{array}{l}\text { mGmUmG mCTC AGG CTA GCT ACA ACG AGG TmGmU mC } \\
\text { mU }\end{array}$ \\
\hline $8 \mathrm{nt} \mathrm{mDz}$ & $\begin{array}{l}\text { mUmG mUmGC TCA GGC TAG CTA CAA CGA GGT G mUmC } \\
\text { mUmU }\end{array}$ \\
\hline $9 \mathrm{nt} \mathrm{mDz}$ & $\begin{array}{l}\text { mCmUmG mUGC TCA GGC TAG CTA CAA CGA GGT GTmC } \\
\text { mUmUmU }\end{array}$ \\
\hline Fluorogenic substrate & FAM-AA AGA CAC CrArU GAG CAC AG-BHQ \\
\hline FAM-labeled substrate & FAM-AA AGA CAC CrArU GAG CAC AG \\
\hline 11nt toehold Dz strand & $\begin{array}{l}\text { TTG CAT TGC AmCmU mGmUG CTC AGG CTA GCT ACA } \\
\text { ACG AGG TGT mCmUmU mU }\end{array}$ \\
\hline Lock strand & GAG CAC AGT GCA ATG CAA CTA CAA TGC AC \\
\hline Inactive Dz strand & $\begin{array}{l}\text { TTG CAT TGC AmGmC mUmGT TAT GGG CTA GCT ACA } \\
\text { ACG ATT CCG mUmGmC mU }\end{array}$ \\
\hline $\begin{array}{l}\text { 11nt toehold Dz strand with } \\
\text { modified } \beta \text { domain }\end{array}$ & $\begin{array}{l}\text { mUmUmG mCmAmU mUmGmC mAmCmU mGmUG CTC } \\
\text { AGG CTA GCT ACA ACG AGG TGT mCmUmU mU }\end{array}$ \\
\hline $\begin{array}{l}\text { Inactive } \mathrm{Dz} \text { strand } \text { with } \\
\text { modified } \beta \text { domain }\end{array}$ & $\begin{array}{l}\text { mUmUmG mCmAmU mUmGmC mAmGmC mUmGT TAT GGG } \\
\text { CTA GCT ACA ACG ATT CCG mUmGmC mU }\end{array}$ \\
\hline $\begin{array}{l}\text { Lock strand with modified } \alpha^{\prime} \text {, } \\
\beta^{\prime} \text { and } \gamma^{\prime} \text { domains }\end{array}$ & $\begin{array}{l}\text { GAG CmAmC mAmGmU mGmCmA mAmUmG mCmAmA } \\
\text { mCmUmA mCmAmA mUmGmC mAmC }\end{array}$ \\
\hline $\begin{array}{l}\text { 11nt toehold inactive } \mathrm{Dz} \\
\text { strand with amine (for } \mathrm{Cy} 5 \\
\text { labeling) }\end{array}$ & $\begin{array}{l}\text { /5AmMC6/TT GCA TTG CAG CTG TTA TGG GCT AGC TAC } \\
\text { AAC GAT TCC GTG CT }\end{array}$ \\
\hline
\end{tabular}




\begin{tabular}{|l|l|}
\hline $\begin{array}{l}\text { Lock strand for inactive Dz } \\
\text { strand (for locked inactive } \\
\text { DzNPs) }\end{array}$ & $\begin{array}{l}\text { ATA ACA GCT GCA ATG CAA CTA CAA TGC ACT TTT TTT } \\
\text { TTT /3ThioMC3-D/ }\end{array}$ \\
\hline $\begin{array}{l}\text { 7nt toehold Dz strand with } \\
\text { amine (for Cy5 labeling) }\end{array}$ & $\begin{array}{l}\text { /5AmMC6/GT AGT TGC ATT GCA mCmUmG mUGC TCA } \\
\text { GGC TAG CTA CAA CGA GGT GTmC mUmUmU }\end{array}$ \\
\hline $\begin{array}{l}\text { 4nt toehold Dz strand with } \\
\text { amine (for Cy5 labeling) }\end{array}$ & $\begin{array}{l}\text { /5AmMC6/AT TGT AGT TGC ATT GCA mCmUmG mUGC TCA } \\
\text { GGC TAG CTA CAA CGA GGT GTmC mUmUmU }\end{array}$ \\
\hline $\begin{array}{l}\text { Lock strand for 11nt, 7nt and } \\
\text { 4nt Dz strand (for locked } \\
\text { DzNPs with toehold) }\end{array}$ & $\begin{array}{l}\text { GAG CAC AGT GCA ATG CAA CTA CAA TGC ACT TTT TTT } \\
\text { TTT /3ThioMC3-D/ }\end{array}$ \\
\hline $\begin{array}{l}\text { Lock strand without toehold } \\
\text { for locked DzNPs without } \\
\text { toehold) }\end{array}$ & GAG CAC AGT GCA ATG CAA TTT TTT TTT T/3ThioMC3-D/ \\
\hline $\begin{array}{l}\text { Locked strand with } \\
\text { scrambled toehold (for locked } \\
\text { DzNPs with scrambled } \\
\text { toehold) }\end{array}$ & $\begin{array}{l}\text { GAG CAC AGT GCA ATG CAA GTA ACA TCT TTT TTT TTT } \\
\text { /3ThioMC3-D/ }\end{array}$ \\
\hline $\begin{array}{l}\text { Dz strand for Dz anchored } \\
\text { locked DzNPs }\end{array}$ & $\begin{array}{l}\text { /5ThioMC6-D/TT TTT TTT TTT TTT TTT TTT TTG CAT TGC } \\
\text { AmCmU mGmUG CTC AGG CTA GCT ACA ACG AGG TGT } \\
\text { mCmUmU mU }\end{array}$ \\
\hline $\begin{array}{l}\text { Lock strand for Dz anchored } \\
\text { locked DzNPs with amine } \\
\text { (for Cy5 labeling) }\end{array}$ & GAG CAC AGT GCA ATG CAA CTA CAA TGC AC/3AmMO/ \\
\hline
\end{tabular}


Table S2. Library of mouse TNF $\alpha$ DNAzymes screened

$\mathrm{m}=2$ '-O-Methyl modification; 3InvdT=3' Inverted dT

\begin{tabular}{|c|c|}
\hline Name & Sequence $\left(5^{\prime} \rightarrow 3^{\prime}\right)$ \\
\hline NS Dz & TCA AGG GAG GCT AGC TAC AAC GAA AGA AGC GG/3InvdT/ \\
\hline Dz110 & mGmGmG mACA GAA GGC TAG CTA CAA CGA CTG CmCmU mGmG \\
\hline Dz168 & mUmGmU mGCT CAG GCT AGC TAC AAC GAG GTG TmCmU mUmU \\
\hline Dz540 & $\begin{array}{l}\text { mUmGmA mAGA GAA GGC TAG CTA CAA CGA CTG GGmA } \\
\text { mGmUmA }\end{array}$ \\
\hline Dz591 & mCmGmG mCTG AGG CTA GCT ACA ACG AGG TGmU mGmGmG \\
\hline Dz705 & mCmCmA mGGT ATA GGC TAG CTA CAA CGA GGG CTmC mAmUmA \\
\hline Dz1248 & mCmAmA mATA TAA AGG CTA GCT ACA ACG AAG AGmG mGmGmG \\
\hline Dz1252 & $\begin{array}{l}\text { mGmUmG mCAA ATA GGC TAG CTA CAA CGA AAA TAG mAmGmG } \\
\text { mG }\end{array}$ \\
\hline Dz1254 & $\begin{array}{l}\text { mAmAmG mUGC AAA GGC TAG CTA CAA CGA ATA AAT AmGmA } \\
\text { mGmG }\end{array}$ \\
\hline
\end{tabular}


Table S3. Primer sequences

\begin{tabular}{|l|l|}
\hline Primer & Sequence $\left(\mathbf{5}^{\prime} \rightarrow \mathbf{3}{ }^{\prime}\right)$ \\
\hline TNF $\alpha \_F o r w a r d$ & CCAGAACATCTTGGAAATAGCTC \\
\hline TNF $\alpha \_$Reverse & GGACCGATCACCCCGAAGT \\
\hline 18 s_Forward & AGGAATTGACGGAAGGGCACCA \\
\hline 18 s_Reverse & GTGCAGCCCCGGACATCTAAG \\
\hline
\end{tabular}

\section{Reference}

1. Sugimoto, N.; Nakano, S. I.; Yoneyama, M.; Honda, K. I. Improved Thermodynamic Parameters and Helix Initiation Factor to Predict Stability of DNA Duplexes. Nucleic Acids Research 1996, 24 (22), 4501-4505. 